Research article

Open Access

\title{
RANKL increases the level of Mcl-1 in osteoclasts and reduces bisphosphonate-induced osteoclast apoptosis in vitro
}

\author{
Karen A Sutherland*, Helena L Rogers*, Denise Tosh and Michael J Rogers
}

\author{
Bone \& Musculoskeletal Research Programme, School of Medicine \& Dentistry, Institute of Medical Sciences, University of Aberdeen, Foresterhill, \\ Aberdeen, AB25 2ZD, UK \\ * Contributed equally
}

Corresponding author: Michael J Rogers, m.j.rogers@abdn.ac.uk

Received: 25 Jun 2008 Revisions requested: 31 Jul 2008 Revisions received: 8 Apr 2009 Accepted: 30 Apr 2009 Published: 30 Apr 2009

Arthritis Research \& Therapy 2009, 11:R58 (doi:10.1186/ar2681)

This article is online at: http://arthritis-research.com/content/11/2/R58

(c) 2009 Sutherland et al.; licensee BioMed Central Ltd.

This is an open access article distributed under the terms of the Creative Commons Attribution License (http://creativecommons.org/licenses/by/2.0), which permits unrestricted use, distribution, and reproduction in any medium, provided the original work is properly cited.

\begin{abstract}
Introduction Bisphosphonates are the most widely used class of drug for inhibiting osteoclast-mediated bone loss, but their effectiveness at preventing joint destruction in rheumatoid arthritis has generally been disappointing. We examined whether the ability of bisphosphonates to induce osteoclast apoptosis and inhibit bone resorption in vitro is influenced by the cytokine receptor activator of nuclear factor-kappa B ligand (RANKL), an important mediator of inflammation-induced bone loss.

Methods Rabbit osteoclasts were treated with the bisphosphonates clodronate or alendronate for up to 48 hours in the absence or presence of RANKL. Changes in cell morphology and induction of apoptosis were examined by scanning electron microscopy, whilst resorptive activity was determined by measuring the area of resorption cavities. Changes in the level of anti-apoptotic proteins, including Mcl-1, $\mathrm{Bcl}-2$, and $\mathrm{Bcl}-\mathrm{x}_{>\mathrm{L}}$, were determined in rabbit osteoclasts and in cytokine-starved mouse osteoclasts by Western blotting.

Results RANKL significantly attenuated the ability of both clodronate and alendronate to induce osteoclast apoptosis and inhibit bone resorption. Treatment of rabbit osteoclasts with RANKL was associated with an increase in the anti-apoptotic protein $\mathrm{Mcl}-1$ but not $\mathrm{Bcl}-2$. A role for $\mathrm{Mcl}-1$ in osteoclast survival was suggested using osteoclasts generated from mouse bone marrow macrophages in the presence of RANKL + macrophage colony-stimulating factor (M-CSF) since cytokine deprivation of mouse osteoclasts caused a rapid loss of Mcl-1 (but not Bcl-2 or Bcl- $\mathrm{x}_{\mathrm{L}}$ ), which preceded the biochemical and morphological changes associated with apoptosis. Loss of Mcl1 from mouse osteoclasts could be prevented by factors known to promote osteoclast survival (RANKL, M-CSF, tumour necrosis factor-alpha [TNF- $\alpha$ ], or lipopolysaccharide [LPS]).

Conclusions RANKL protects osteoclasts from the apoptosisinducing and anti-resorptive effects of bisphosphonates in vitro. The ability of RANKL (and other pro-inflammatory factors such as TNF- $\alpha$ and LPS) to increase the level of Mcl-1 in osteoclasts may explain the lack of effectiveness of some bisphosphonates in preventing inflammation-induced bone loss.
\end{abstract}

\section{Introduction}

The molecular mechanisms by which bisphosphonate (BP) drugs inhibit osteoclast-mediated bone resorption have been clarified in recent years [1]. After targeting bone mineral and internalisation by osteoclasts, simple BPs such as clodronate are metabolised intracellularly by osteoclasts to form nonhydrolysable analogues of ATP which induce osteoclast apoptosis [2]. By contrast, the nitrogen-containing BPs such as alendronate and zoledronate do not appear to be metabolised but are potent inhibitors of farnesyl diphosphate (FPP) synthase, thereby preventing the post-translational prenylation of small GTPases that are necessary for osteoclast polarisation, bone resorption, and cell survival $[3,4]$. Both simple BPs and nitrogen-containing BPs are therefore capable of causing osteoclast apoptosis, in vitro and in vivo [5], but by different molecular mechanisms. The regulation of osteoclast apoptosis

Q-MEM: alpha-minimum essential medium; ALN: 4-amino-1-hydroxy-butylidene-1,1-bisphosphonate (alendronate); BP: bisphosphonate; CLO: dichloromethylene-1,1-bisphosphonate (clodronate); DAPI: 4,6-diamidino-2-phenylindole; EM: electron microscopy; FCS: fetal calf serum; FPP: farnesyl diphosphate; LPS: lipopolysaccharide; MAPK: mitogen-activated protein kinase; M-CSF: macrophage colony-stimulating factor; mTOR: mammalian target of rapamycin; PBS: phosphate-buffered saline; RA: rheumatoid arthritis; RANKL: receptor activator of nuclear factor-kappa-B ligand; TNF- $\alpha$ : tumour necrosis factor-alpha; TRAP: tartrate-resistant acid phosphatase. 
appears to be an important mechanism of physiological bone homeostasis since a variety of growth factors and cytokines that stimulate bone resorption (such as receptor activator of nuclear factor-kappa B ligand [RANKL], interleukin-1, and tumour necrosis factor-alpha [TNF- $\alpha$ ]) also prevent osteoclast apoptosis (reviewed elsewhere $[6,7]$ ). In this study, we examined the extent to which RANKL might antagonise the antiresorptive activity of clodronate and alendronate in vitro. This is of particular relevance in the context of rheumatoid arthritis (RA), in which high levels of RANKL expressed by synovial fibroblasts and $\mathrm{T}$ lymphocytes contribute to osteoclast-mediated joint destruction [8-10]. Some BPs have been shown to prevent local and systemic bone loss in some animal models of inflammation-induced arthritis [11-14] and to preserve joint architecture in a recent clinical trial [15]. However, the effectiveness of BPs at preventing joint destruction in other clinical studies in patients with RA has been disappointing [16-19]. The reasons for this are not completely clear but could involve factors in the local environment of the inflamed joint, such as RANKL, that might antagonise the anti-resorptive action of BPs.

\section{Materials and methods Reagents}

Clodronate (dichloromethylene-1,1-bisphosphonate) (CLO) and alendronate (4-amino-1-hydroxy-butylidene-1,1-bisphosphonate) (ALN) were kindly provided by Procter \& Gamble Pharmaceuticals (Cincinnati, OH, USA). Stock solutions were prepared in phosphate-buffered saline (PBS) (the $\mathrm{pH}$ adjusted to $\mathrm{pH} 7.4$ with $5 \mathrm{M}$ sodium hydroxide) and filter-sterilised prior to use. Cell culture reagents were from Sigma-Aldrich (Poole, UK).

\section{Quantification of osteoclast apoptosis}

Mature osteoclasts were isolated from rabbit long bones and seeded into 24-well plates as previously described [3]. The following day, the plates were washed several times with PBS to remove the majority of stromal cells, leaving cultures of approximately 95\% pure osteoclasts (tartrate-resistant acid phosphatase [TRAP]-positive multinucleated cells). Cultures were incubated with alpha-minimum essential medium ( $\alpha$ MEM) containing 10\% (vol/vol) fetal calf serum (FCS), $100 \mathrm{U} /$ $\mathrm{mL}$ penicillin, $100 \mu \mathrm{g} / \mathrm{mL}$ streptomycin, and $100 \mu \mathrm{M}$ CLO or ALN in the absence or presence of $100 \mathrm{ng} / \mathrm{mL}$ recombinant human RANKL (PeproTech, Rocky Hill, NJ, USA) (three wells per treatment). After 48 hours, the culture media were removed and adherent cells were fixed with $4 \%$ formaldehyde and either stained with $1 \mu \mathrm{g} / \mathrm{mL}$ 4,6-diamidino-2-phenylindole (DAPI) in PBS or stained for TRAP [20]. The number of TRAPpositive multinucleated osteoclasts per well or the proportion of osteoclasts with DAPI-stained nuclei showing characteristic apoptotic nuclear morphology (chromatin condensation and nuclear fragmentation) [21] was determined using a Zeiss Axiovert 135 microscope and $\times 20$ objective (Carl Zeiss, Jena, Germany).

\section{Analysis of osteoclast morphology by scanning electron microscopy}

Bone marrow cells from rabbit long bones were seeded onto discs of elephant ivory in 96-well plates [20] and cultured with $\alpha$-MEM containing $10 \%$ (vol/vol) FCS with $50 \mu \mathrm{M}$ CLO or ALN in the absence or presence of $100 \mathrm{ng} / \mathrm{mL}$ recombinant human RANKL. After 24 hours, cells were fixed in 2.5\% (vol/ vol) glutaraldehyde and $2.5 \mathrm{mM} \mathrm{MgCl}_{2}$ in $0.089 \mathrm{M}$ phosphate buffer ( $\mathrm{pH}$ 7.2) for 3 hours at room temperature. Discs were washed overnight in $0.1 \mathrm{M}$ phosphate buffer ( $\mathrm{pH} 7.2$ ), postfixed in osmium tetroxide for 1 hour, washed in distilled water, and dehydrated through a graded series of ethanol solutions. The samples were critical-point-dried from $\mathrm{CO}_{2}$, glued onto aluminium stubs with colloidal silver adhesive, sputter-coated with $20 \mathrm{~nm}$ platinum, and examined in a Jeol JSM-35CF scanning electron microscope (EM) (Jeol Ltd., Tokyo, Japan) operating at $10 \mathrm{kV}$.

\section{Quantification of osteoclast-mediated bone resorption}

Rabbit bone marrow cells were seeded onto ivory discs as described above and cultured with $\alpha$-MEM containing $10 \%$ (vol/vol) FCS with $100 \mu \mathrm{M} \mathrm{CLO}$ or ALN in the absence or presence of $100 \mathrm{ng} / \mathrm{mL}$ recombinant human RANKL (four wells per treatment). After 48 hours, the media were removed, cells were wiped from the ivory discs, and the total area of mineral resorbed per disc was determined using a reflected light microscope [3].

\section{Measurement of caspase-9 activity in osteoclasts}

Rabbit osteoclasts, purified as described above, were cultured with $\alpha$-MEM containing $100 \mu \mathrm{M} \mathrm{ALN} \pm 100 \mathrm{ng} / \mathrm{mL}$ RANKL for 48 hours. Unfixed, adherent cells were stained using an Apofluor Green Caspase Activity Assay kit (Enzyme Systems Products, Livermore, CA, USA). This involves the covalent binding of a fluorescently labelled, cell-permeable caspase inhibitor to active caspase-9, thus allowing the detection of cells with caspase- 9 activity. Cells were counterstained with Hoechst 33342, washed to remove excess stain, and visualised using a Zeiss Axiovert 135 microscope and $\times 20$ objective.

\section{Western blot analysis}

Mature osteoclasts were isolated from rabbit long bones, seeded into 10-cm-diameter Petri dishes, and purified as previously described [3]. Purified osteoclasts were cultured for 48 hours with $100 \mathrm{ng} / \mathrm{mL}$ RANKL or with $100 \mu \mathrm{M} \mathrm{ALN} \pm 100$ $\mathrm{ng} / \mathrm{mL}$ RANKL (four dishes per treatment). Dishes were rinsed with PBS, and osteoclasts were lysed in $300 \mu \mathrm{L}$ of RIPA buffer (1\% [vol/vol] NP-40, 0.5\% [wt/vol] sodium deoxycholate, and $0.1 \%$ [wt/vol] SDS) containing $20 \mu \mathrm{L}$ of Sigma-Aldrich protease inhibitor cocktail (P-8340). Protein $(40 \mu \mathrm{g})$ from each sample was electrophoresed under reducing conditions on a $12.5 \%$ polyacrylamide/SDS gel and then transferred onto polyvinyldifluoride membrane. Blots were hybridised with goat polyclonal anti-Rap1A (sc1482; Santa Cruz Biotechnology, 
Inc., Santa Cruz, CA, USA), rabbit polyclonal anti-Mcl-1 or mouse monoclonal anti-Bcl-2 (Santa Cruz Biotechnology, Inc.), anti- $\beta$-actin (Sigma-Aldrich), or rabbit polyclonal antibodies to cIAP-1, XIAP, or clAP-2 (R\&D Systems, Inc., Minneapolis, MN, USA), followed by horseradish peroxidise-conjugated secondary antibodies. Blots were visualised after chemiluminescence detection using a Bio-Rad FluorS Max imager (BioRad Laboratories, Inc., Hercules, CA, USA).

\section{Generation and cytokine starvation of mouse osteoclasts}

Mouse osteoclasts were generated in vitro from macrophage colony-stimulating factor (M-CSF)-dependent bone marrow macrophages. Bone marrow cells were flushed into $10-\mathrm{cm}$ Petri dishes (Falcon, now part of BD Biosciences, San Jose, CA, USA) from the tibiae and femorae of adult male C57BL/6 mice and cultured in $\alpha-M E M$ containing $100 \mathrm{U} / \mathrm{mL}$ penicillin, $100 \mu \mathrm{g} / \mathrm{mL}$ streptomycin, $1 \mathrm{mM}$ glutamine, 10\% FCS, and $100 \mathrm{ng} / \mathrm{mL}$ murine M-CSF (R\&D Systems, Inc.). After 2 days, non-adherent cells were removed and the adherent cells were re-seeded into 24-well plates (Corning Life Sciences, Acton, MA, USA) at a density of $2.5 \times 10^{4}$ cells per well in the medium described above containing $25 \mathrm{ng} / \mathrm{mL}$ M-CSF and $20 \mathrm{ng} / \mathrm{mL}$ murine RANKL (R\&D Systems, Inc.). Multinucleated, TRAPpositive osteoclasts formed after 5 days.

These cultures are amenable to studies on osteoclast survival since (unlike rabbit osteoclasts) the cells are highly dependent on the presence of exogenous pro-survival factors such as $\mathrm{M}$ CSF, RANKL, TNF- $\alpha$, or lipopolysaccharide (LPS). To induce osteoclast apoptosis, the medium was removed and replaced with fresh medium lacking M-CSF/RANKL or with medium containing $100 \mathrm{ng} / \mathrm{mL}$ M-CSF, $100 \mathrm{ng} / \mathrm{mL}$ RANKL, $10 \mathrm{ng} / \mathrm{mL}$ TNF- $\alpha, 0.1 \mu \mathrm{M}$ LPS, or M-CSF + RANKL (control). After 2 to 12 hours, cell lysates were analysed by Western blotting as described above using antibodies to $\mathrm{Mcl}-1, \mathrm{Bcl}-2, \mathrm{Bcl}-\mathrm{x}_{\mathrm{L}}$ (Santa Cruz Biotechnology, Inc.), and cleaved caspase-3 (Promega Corporation, Madison, WI, USA). After 8 hours of cytokine starvation, osteoclasts grown on glass coverslips were also fixed and processed for analysis by scanning EM as described above.

\section{Statistical analysis}

The effects of BPs and RANKL on osteoclast number, osteoclast apoptosis, bone resorption, and caspase- 9 activity were analysed by analysis of variance with a Bonferroni post hoc test (SPSS version 9.0; SPSS Inc., Chicago, IL, USA).

\section{Results \\ RANKL attenuates osteoclast apoptosis induced by clodronate or alendronate}

Treatment with $100 \mu \mathrm{M}$ ALN or CLO reduced the number of adherent osteoclasts (TRAP-positive cells with at least three nuclei) in plastic culture dishes to approximately $52 \%$ and $57 \%$ of control cultures, respectively (Figure 1a). In the pres-
Figure 1
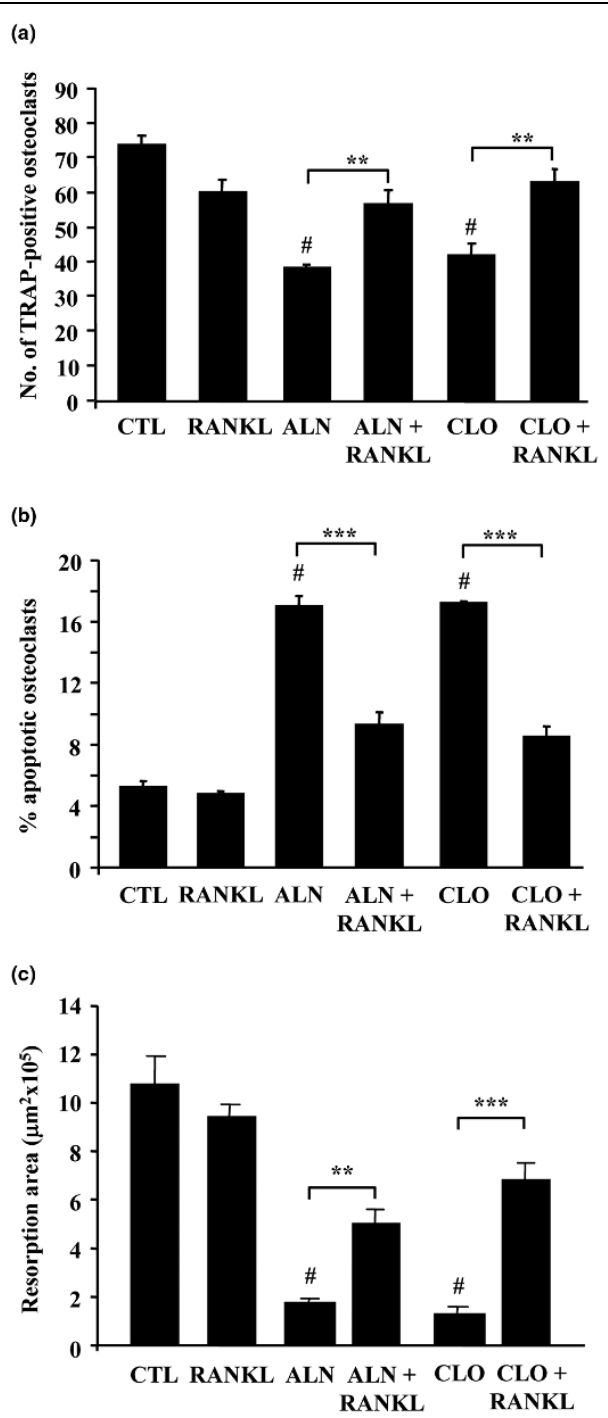

RANKL attenuates the effect of bisphosphonates on osteoclast number, apoptosis, and bone resorption in vitro. Cultures of mature osteoclasts from rabbit bones were treated with $100 \mu \mathrm{M}$ ALN or CLO, $\pm 50 \mathrm{ng} / \mathrm{mL}$ RANKL for 48 hours. Cells were then fixed, stained for tartrate-resistant acid phosphatase (TRAP), and counterstained with DAPI. (a) Results are the mean number of TRAP-positive multinucleated osteoclasts (more than three nuclei per cell) \pm standard error of the mean (SEM) $(n=3)$ or $(b)$ the percentage of non-apoptotic and apoptotic osteoclasts. Data are expressed as the mean \pm SEM $(n=3$ replicates). ${ }^{\star \star} P=0.01,{ }^{\star \star \star} P=0.001$ compared with ALN or CLO alone (analysis of variance). \#Treatment with ALN or CLO alone caused a significant decrease in osteoclast number compared with control (CTL) cultures $(P=0.01)$ and a significant increase in osteoclast apoptosis compared with control cultures $(P=0.001)$. (c) Values of resorption area are the mean resorbed area $\left(\mathrm{mm}^{2}\right)$ per slice \pm SEM $(n=6$ slices $)$. ${ }^{\star \star *} P=0.001$ compared with CLO alone and ${ }^{* \star} P=0.01$ compared with ALN alone (analysis of variance). \#Treatment with ALN or CLO alone caused a significant decrease in osteoclastic bone resorption compared with control cultures $(P=0.001)$. The data shown are representative of three independent experiments. ALN, 4-amino-1-hydroxybutylidene-1,1-bisphosphonate (alendronate); CLO, dichloromethylene-1,1-bisphosphonate (clodronate); DAPI, 4,6-diamidino-2-phenylindole; RANKL, receptor activator of nuclear factor-kappa-B ligand. 

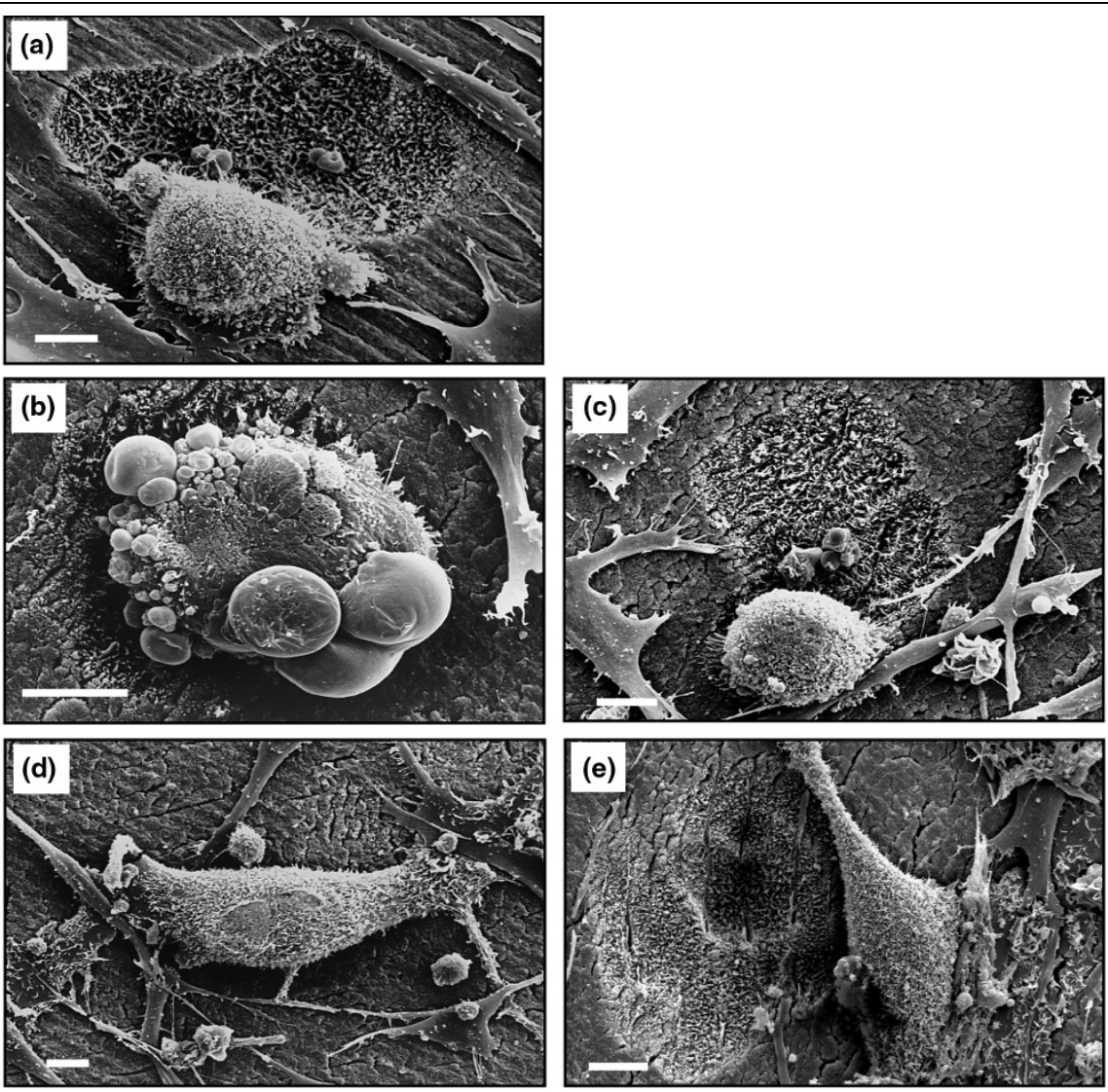

Scanning EM analysis of the effect of CLO, ALN, and RANKL on the morphology of mature osteoclasts cultured in vitro. Rabbit osteoclasts were cultured on ivory discs for 24 hours with $50 \mu \mathrm{M}$ CLO or $50 \mu \mathrm{M}$ ALN, $\pm 100 \mathrm{ng} / \mathrm{mL}$ RANKL. (a) Control. (b) CLO. (c) CLO + RANKL. (d) ALN. (e) $A L N+R A N K L$. Osteoclasts were fixed and processed for scanning EM analysis. Bars $=10 \mu \mathrm{m}$. ALN, 4-amino-1-hydroxy-butylidene-1,1-bisphosphonate (alendronate); CLO, dichloromethylene-1,1-bisphosphonate (clodronate); RANKL, receptor activator of nuclear factor-kappa-B ligand.

ence of $50 \mathrm{ng} / \mathrm{mL}$ RANKL, the reduction in osteoclast number was significantly attenuated (to approximately $77 \%$ and $85 \%$ of control cultures, respectively) $(P<0.01)$.

Osteoclasts in culture dishes that were undergoing apoptosis but remained adherent were identified on the basis of characteristic morphological features (chromatin condensation and nuclear fragmentation) after staining with DAPI [21]. Approximately $17 \%$ of adherent osteoclasts in culture dishes were apoptotic after treatment with $100 \mu \mathrm{M}$ ALN or CLO for 48 hours. This was significantly reduced (to approximately $9 \%$ ) in the presence of $50 \mathrm{ng} / \mathrm{mL}$ RANKL $(P<0.001)$, similar to the proportion of osteoclasts undergoing apoptosis in cultures without BPs (Figure 1b). RANKL alone did not significantly alter the number of adherent osteoclasts or the proportion of apoptotic osteoclasts in cultures in the absence of BPs.

\section{RANKL protects osteoclasts from the anti-resorptive effects of bisphosphonates}

The effect of RANKL on the morphology of BP-treated rabbit osteoclasts was also studied by scanning EM. After 24 hours of culture on ivory discs, $71 \%$ of osteoclasts in control cultures were spread on the mineral surface, often located in or adjacent to extensive and deep resorption cavities (Figure 2a). However, after treatment with $50 \mu \mathrm{M}$ CLO for 24 hours, few, shallow resorption pits were present and $50 \%$ of the osteoclasts were rounded and lacked areas of spreading. Many of these rounded osteoclasts lacked membrane ruffles or microvilli but contained numerous blebs, indicative of cells undergoing apoptosis (Figure 2b). The morphology of other cell types, such as stromal cells, in the culture did not appear to be affected by CLO. The appearance of apoptotic osteoclasts was prevented by the presence of RANKL since 30\% of osteoclasts were rounded when cultured with CLO + RANKL, few of these had membrane blebbing, and $70 \%$ of the osteoclasts appeared similar to those in control cultures, associated with numerous resorption pits (Figure 2c).

Treatment with $50 \mu \mathrm{M}$ ALN for 24 hours caused the appearance of osteoclasts that (although still spread and adherent to the mineral) appeared retracted, with long cell processes (Figure $2 \mathrm{~d}$ ), and were associated (if at all) with only minor resorption pits. These osteoclasts often lacked microvilli but exhibited ridges on the basolateral membrane, and few $(<5 \%)$ 
Figure 3

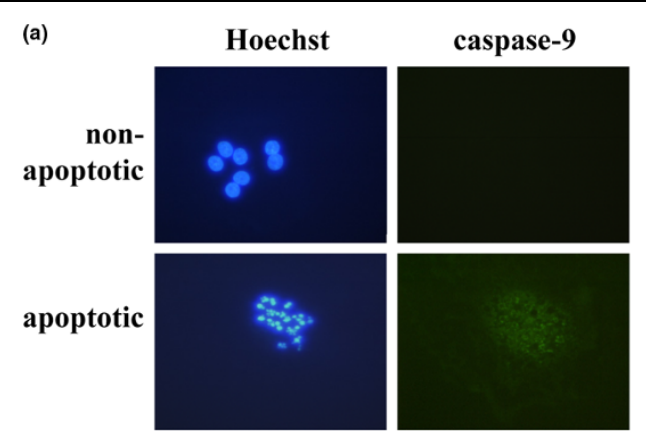

(b)

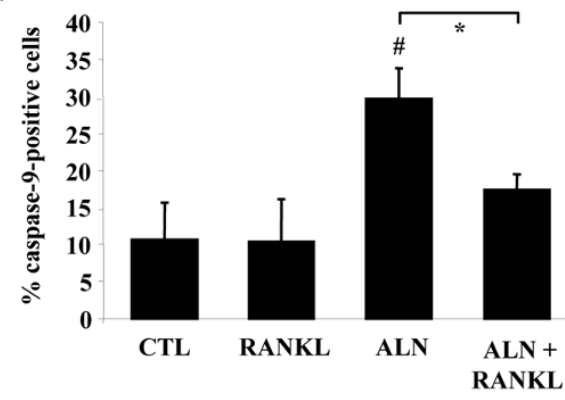

(c)

ALN +

CTL RANKL ALN RANKL

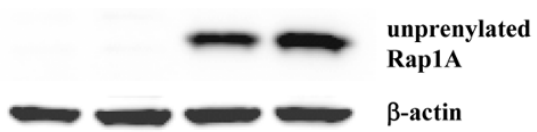

(d)

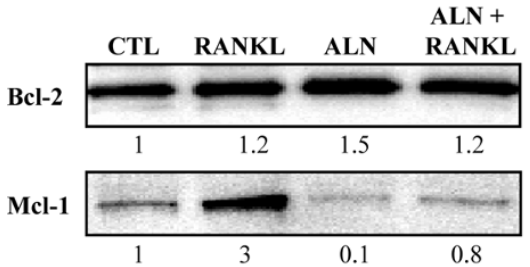

RANKL prevents activation of caspase- 9 and increases $\mathrm{Mcl}-1$ in osteoclasts but does not prevent inhibition of protein prenylation. Cultures of mature osteoclasts from rabbit bones were treated with $100 \mu \mathrm{M} \mathrm{ALN} \pm$ $100 \mathrm{ng} / \mathrm{mL}$ RANKL for 48 hours and stained using an Apofluor Green Caspase-9 Activity Assay kit and Hoechst 33342. (a) A representative non-apoptotic and apoptotic osteoclast. (b) Quantification of caspase9-positive osteoclasts after treatment with alendronate \pm RANKL for 48 hours. ${ }^{*} P \leq 0.05$ compared with ALN alone or ${ }^{\#} P \leq 0.05$ compared with control (CTL) (analysis of variance). Values are the mean \pm standard error of the mean ( $n=3$ replicates) (100 to 150 cells counted per well). The data shown are representative of three independent experiments. (c) Purified rabbit osteoclasts were treated for 48 hours with $100 \mu \mathrm{M}$ ALN $\pm 100 \mathrm{ng} / \mathrm{mL}$ RANKL or with RANKL alone. Cell lysates were then analysed by Western blotting for the unprenylated form of Rap $1 \mathrm{~A}$ and for $\beta$-actin. (d) Purified rabbit osteoclasts were treated for 48 hours with $100 \mu \mathrm{M}$ ALN $\pm 100 \mathrm{ng} / \mathrm{mL}$ RANKL or with $100 \mathrm{ng} / \mathrm{mL}$ RANKL alone. Cell lysates were then analysed by Western blotting for $\mathrm{Mcl}-1$ and $\mathrm{Bcl}-2$. The level of $\mathrm{Mcl}-2$ or $\mathrm{Bcl}-2$ was quantified by densitometric analysis and expressed as a ratio of the level in control cells. Data shown are representative of three independent experiments. ALN, 4-amino-1-hydroxy-butylidene-1,1-bisphosphonate (alendronate); RANKL, receptor activator of nuclear factor-kappa-B ligand. apoptotic osteoclasts were observed with the obvious membrane blebbing observed in CLO-treated cultures. After culture with ALN + RANKL, osteoclasts were mostly well spread with membrane ruffles and surface microvilli, and resorption cavities were more evident (Figure 2e). Some osteoclasts also retained the presence of membrane ridges on the basolateral surface, but few $(<5 \%)$ had the retracted morphology of ALNtreated cells or the blebbed morphology of CLO-treated cells. As expected, when the area of bone resorption was quantified, $100 \mu \mathrm{M}$ ALN or CLO significantly inhibited the resorptive activity of rabbit osteoclasts cultured on ivory discs in vitro. However, the inhibitory effect of ALN or CLO on bone resorption was significantly overcome by the presence of $100 \mathrm{ng} / \mathrm{mL}$ RANKL (Figure 1c).

\section{RANKL reduces caspase-9 activity in osteoclasts}

A fluorescent, cell-permeable caspase- 9 inhibitor was used to identify single cells with caspase- 9 activity (Figure 3a). In control cultures of purified rabbit osteoclasts, approximately $10 \%$ of the cells (Figure $3 b$ ) had detectable caspase- 9 activity (similar to the proportion of cells in control cultures that were identified as apoptotic on the basis of nuclear morphology) (Figure 1b). After treatment with $100 \mu \mathrm{M} \mathrm{ALN}$ for 48 hours, the proportion of caspase-9-positive osteoclasts increased significantly (to about $30 \%$ ). This was significantly reduced, almost to the proportion in control cultures, in the presence of 100 $\mathrm{ng} / \mathrm{mL}$ RANKL.

\section{RANKL does not prevent accumulation of unprenylated Rap1A in osteoclasts}

In accord with our previous studies, treatment of purified rabbit osteoclasts with $100 \mu \mathrm{M}$ ALN for 48 hours caused the accumulation of the unprenylated form of the small GTPase Rap1A, thereby demonstrating that ALN inhibits protein prenylation in osteoclasts [2,22]. Incubation of osteoclasts with $100 \mu \mathrm{M}$ ALN in the presence of $100 \mathrm{ng} / \mathrm{mL}$ RANKL for 48 hours did not prevent the accumulation of unprenylated Rap1A (Figure 3c).

RANKL increases the level of $\mathrm{Mcl}-1$ in rabbit osteoclasts Western blot analysis of purified rabbit osteoclasts showed that treatment with $100 \mathrm{ng} / \mathrm{mL}$ RANKL, $100 \mu \mathrm{M}$ ALN, or ALN + RANKL had no effect on the level of Bcl-2 protein (Figure 3d). However, RANKL alone consistently caused a threefold increase in the level of Mcl-1 protein in osteoclasts. Treatment with ALN caused a decrease of approximately $90 \%$ in $\mathrm{Mcl}-1$, although co-treatment with RANKL almost completely prevented this effect and maintained the level of $\mathrm{Mcl}-1$ similar to that in control cells (Figure $3 d$ ).

\section{Loss of $\mathrm{Mcl}-1$ precedes apoptosis during cytokine deprivation of mouse osteoclasts but is prevented by pro-survival factors}

To further examine the importance of $\mathrm{Mcl}-1$ in osteoclast survival, multinucleated osteoclasts were generated from M-CSF- 
(a)
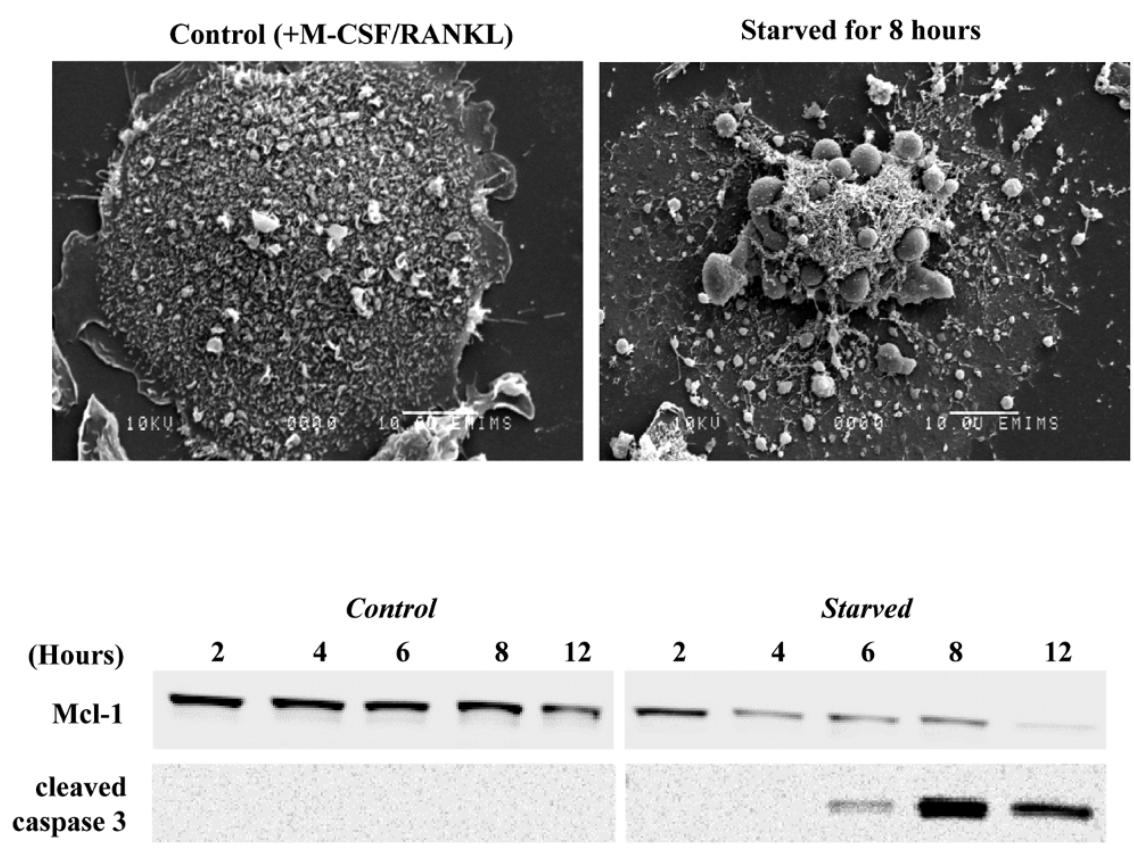

(b)
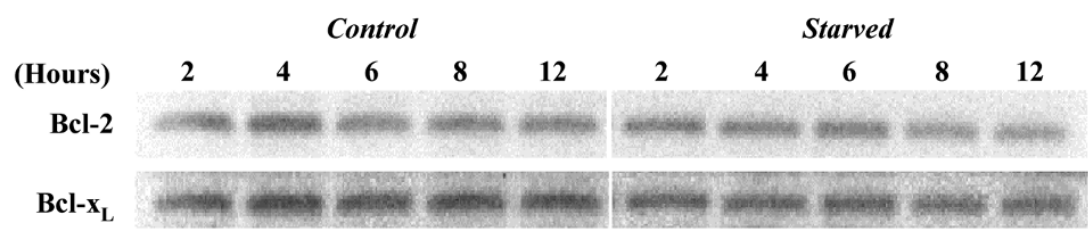

(c)

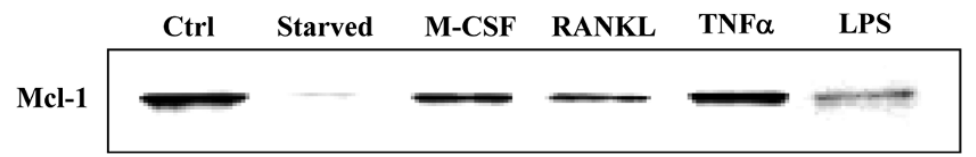

Mcl-1 levels decrease rapidly in mouse osteoclasts following cytokine starvation but are restored by pro-survival factors. Mouse osteoclasts were generated by culturing bone marrow macrophages for 5 days with macrophage colony-stimulating factor (M-CSF) + receptor activator of nuclear factor-kappa-B ligand (RANKL). (a) Osteoclasts were starved of M-CSF and RANKL for 8 hours and then fixed and processed for scanning EM analysis. Representative osteoclasts from non-starved (control) or starved cultures are shown. Bars $=10 \mu \mathrm{m}$. (b) Mouse osteoclasts were starved of MCSF and RANKL for 2 to 12 hours. Western blot analysis was then used to determine the level of Mcl-1, cleaved caspase-3, Bcl-2, and Bcl- $x_{L}$ at each time point. (c) After osteoclasts were generated, the medium was replaced with normal medium (control [Ctrl]: M-CSF + RANKL), with medium lacking cytokines (starved), or with medium containing recombinant M-CSF, RANKL, tumour necrosis factor-alpha (TNF- $\alpha$ ), or lipopolysaccharide (LPS). After 12 hours, Western blotting was used to determine the level of Mcl-1 in $40 \mu \mathrm{g}$ of cell lysate. Data shown are representative of three independent experiments.

dependent mouse bone marrow macrophages by culturing the latter cells for 5 days with M-CSF + RANKL. When the osteoclasts were starved of these cytokines, morphological changes indicative of apoptosis (Figure 4a) were apparent after 6 to 8 hours, consistent with the appearance in Western blots of the cleaved form of caspase-3 after 6 hours of cytokine starvation (Figure 4b). The appearance of apoptotic osteoclasts and cleaved caspase- 3 was preceded by a decrease in the level of Mcl-1 (noticeable after 4 hours). Mcl1 was almost completely absent after 12 hours of cytokine starvation, although the levels of $\mathrm{Bcl}-1$ and $\mathrm{Bcl}-\mathrm{x}_{\mathrm{L}}$ did not change during this time (Figure 4b). The loss of Mcl-1 that occurred in mouse osteoclasts following cytokine starvation could be prevented by the addition of M-CSF, RANKL, TNF- $\alpha$, or LPS (Figure 4c).

\section{Discussion}

BPs have become the mainstay of treatment for post-menopausal osteoporosis, Paget disease, and tumour-associated osteolysis and have been shown to prevent generalised bone loss in patients with RA treated with corticosteroids (reviewed recently by Breuil and Euller-Ziegler [16]). However, apart 
from a recent clinical study using the highly potent BP zoledronic acid in patients with RA [15] and two studies of zoledronic acid in animal models of RA $[12,13]$, the effectiveness of BPs at preventing focal bone loss has been less convincing [16-19]. It has recently been suggested that the reason for this relative lack of effect on local, inflammatory bone loss is due to factors in the inflamed joint, such as TNF$\alpha$, that antagonise the ability of BPs to inhibit osteoclasts. Zhang and colleagues [23], using TNF- $\alpha$ transgenic mice, showed that $\mathrm{Bcl}-\mathrm{x}_{\mathrm{L}}$ levels were markedly higher in osteoclasts, an effect that appeared to be caused by TNF- $\alpha$-induced expression of Ets-2. Furthermore, overexpression of Ets-2 or $\mathrm{Bcl}-\mathrm{x}_{\mathrm{L}}$ protected osteoclasts from $\mathrm{ALN}$-induced apoptosis in vitro. RANKL is also abundant in the rheumatoid microenvironment and drives the enhanced osteoclastogenesis and hence excessive osteoclast-mediated destruction of bone [8-10]. In our study, we demonstrate that RANKL also protects osteoclasts from the apoptosis-inducing and anti-resorptive effects of ALN or CLO in vitro. The number of apoptotic rabbit osteoclasts was significantly lower in cultures treated for 48 hours with ALN or CLO in the presence of RANKL than in cultures treated with the BPs alone. Consistent with this, RANKL preserved the total number of osteoclasts in cultures treated with the BPs and also significantly overcame the anti-resorptive effect of the BPs when osteoclasts were cultured on dentine discs. This ability of RANKL to rescue osteoclasts from the effects of BPs was also observed morphologically by using scanning EM. Treatment of osteoclasts with CLO for 24 hours caused morphological changes associated with apoptotic cell death, consistent with the ability of CLO to induce osteoclast apoptosis via the formation and rapid accumulation of a cytotoxic metabolite $[2,24,25]$. The morphological changes associated with ALN treatment for 24 hours (cell retraction and alterations in plasma membrane morphology) are consistent with the inhibition of FPP synthase. This leads to the inhibition of protein prenylation and the slow accumulation of unprenylated small GTPases such as Rap1A $[1,26]$. The morphological appearance of osteoclasts after 24 hours of treatment with ALN therefore probably indicates cells with altered cytoskeletal arrangement and vesicular trafficking, as well as cells in the very early stages of apoptosis, as a result of abnormal small GTPase signalling [4,27]. In the presence of RANKL, these effects of ALN and CLO on osteoclast morphology were largely overcome, at least over a 24-hour culture period. In the case of ALN, this was not due to any ability of RANKL to prevent inhibition of FPP synthase, since RANKL treatment did not prevent the accumulation of unprenylated Rap1A in osteoclasts.

We have previously shown that BP-induced osteoclast apoptosis involves loss of mitochondrial membrane potential, leading (presumably as a result of subsequent release of cytochrome $\mathrm{C}$ from mitochondria and activation of procaspase-9) to the cleavage and activation of procaspase-3 [21]. To identify a potential mechanism by which RANKL prevents
BP-induced apoptosis, we examined in more detail the events involved in ALN-induced apoptosis. Co-treatment with RANKL prevented the increase in the proportion of osteoclasts with active caspase- 9 seen after ALN treatment, suggesting that RANKL either prevents the mitochondrial changes that lead to activation of procaspase-9 [28] or prevents procaspase-9 activation subsequent to the release of cytochrome $C$ (for example, by increasing the expression of XIAP [29]). Although expression of XIAP and cIAP1/2 can be stimulated via nuclear factor-kappa-B [30], which is a major signalling pathway activated by RANKL [7], we did not observe any effect of RANKL on the level of XIAP, cIAP1, or clAP2 in rabbit osteoclasts by Western blotting (data not shown), consistent with earlier studies on murine osteoclasts [31]. This suggests that RANKL probably prevents apoptosis by preventing mitochondrial changes prior to caspase activation, perhaps via members of the $\mathrm{Bcl}-2$ family of proteins that regulate the mitochondrial pathway of apoptosis [32,33].

RANKL did not alter the level of Bcl-2 in rabbit osteoclasts but caused a marked increase in the level of Mcl-1, an anti-apoptotic member of the $\mathrm{Bcl}-2$ family that is expressed in cells of the myeloid lineage such as macrophages and neutrophils $[34,35]$, and is also known to be increased in synovial macrophages and fibroblasts in RA $[36,37]$ as well as in synovial fluid lymphocytes in juvenile idiopathic arthritis [38]. In myeloid cells, Mcl-1 prevents apoptosis and its expression is highly regulated by survival-promoting factors via the PI3K/Akt/ mammlian target of rapamycin (mTOR)/S6 kinase as well as mitogen-activated protein kinase (MAPK) signalling pathways $[39,40]$. The latter are also known to be activated by M-CSF, TNF- $\alpha$, and RANKL $[7,41]$, which promote osteoclast survival [6]. Whilst our work was in progress, Bradley and colleagues [42] showed that Mcl-1 mRNA and protein are upregulated in osteoclasts by M-CSF via activation of MEK/ERK (MAPK kinase/extracellular regulated kinase) and increased expression of Egr2. Hence, although the exact mechanism by which RANKL upregulates $\mathrm{Mcl}-1$ in osteoclasts remains to be proven, it is highly likely that this also involves MAPK and/or mTOR signalling pathways. ALN treatment alone caused a decrease in $\mathrm{Mcl}-1$ levels, perhaps since the activation of $\mathrm{mTOR} / \mathrm{S} 6$ kinase in osteoclasts appears to involve geranylgeranylated proteins such as Rac [41], and ALN is known to effectively prevent protein geranylgeranylation [1,4]. With myeloma cells, others have also shown that the inhibition of protein geranylgeranylation causes the loss of Mcl-1 [43]. In our study, RANKL restored the level of $\mathrm{Mcl}-1$ to that in control osteoclasts, but this was still less than the level of $\mathrm{Mcl}-1$ in the presence of RANKL alone. Hence, ALN and RANKL may have opposing effects on the same signalling pathways (perhaps involving $\mathrm{mTOR}$ ) that are required for $\mathrm{Mcl}-1$ expression in osteoclasts.

In further support of an important role for $\mathrm{Mcl}-1$ in osteoclast survival, we found that the level of $\mathrm{Mcl}-1$ rapidly decreased fol- 
lowing the removal of M-CSF and RANKL from cultures of mouse osteoclasts, which are highly dependent on the presence of such survival factors. In this model, the loss of Mcl-1 preceded the appearance of morphological and biochemical features of apoptosis and occurred in the absence of changes in the level of Bcl-2 or Bcl- $\mathrm{x}_{\mathrm{L}}$. In addition, the loss of $\mathrm{Mcl}-1$ in mouse osteoclasts could be prevented by the addition of factors (LPS, TNF- $\alpha$, M-CSF, and RANKL) that are known to promote osteoclast survival $[6,7,44]$. The loss of $\mathrm{Mcl}-1$ in osteoclasts probably occurred by proteasomal degradation since we also found that the proteasome inhibitor MG132 caused an increase in the level of $\mathrm{Mcl}-1$ in mouse osteoclasts (data not shown). This is consistent with a requirement in osteoclasts for continual protein synthesis to prevent apoptosis $[41,45]$. Together, these observations indicate that Mcl-1 plays an important role in maintaining osteoclast survival.

Mcl-1 inhibits activation of the mitochondrial pathway of apoptosis by interacting with pro-apoptotic $\mathrm{Bcl}-2$ family proteins such as Bak and Bim, thereby preventing the increased mitochondrial permeability that leads to caspase activation $[33,46,47]$. Lack of Bim leads to enhanced osteoclast survival and the pro-survival factor M-CSF decreases Bim levels in osteoclasts and osteoclast precursors $[48,49]$, probably by increasing ubiquitin-dependent degradation of Bim via upregulation of $\mathrm{cCbl}$ [42]. The role of Bim in BP-induced osteoclast apoptosis is not known, although a recent study showed that apoptosis of MCF-7 breast cancer cells induced by the BP risedronate involved increased levels of Bim [50]. Upregulation of Mcl-1 (and therefore antagonism of Bim) by RANKL is therefore a likely mechanism by which RANKL prevents BPinduced apoptosis of osteoclasts.

\section{Conclusions}

We have shown that RANKL protects osteoclasts from the pro-apoptotic and hence anti-resorptive effects of the BPs CLO and ALN in vitro. This protective effect of RANKL is likely to be mediated at least in part by an increase in the level of the anti-apoptotic protein Mcl-1. The ability of RANKL, together with other factors such as TNF- $\alpha$ [23], to rescue osteoclasts from the pro-apoptotic effects of BPs may account for the apparent lack of effectiveness of BPs (particularly the less potent BPs such as CLO and ALN) in preventing local, inflammation-induced bone loss in RA.

\section{Competing interests}

MJR has received research funding from Procter \& Gamble (Cincinnati, OH, USA), Novartis (Basel, Switzerland), and Roche (Basel, Switzerland) and acts as a consultant for Novartis and Procter \& Gamble. The other authors declare that they have no competing interests.

\section{Authors' contributions}

MJR conceived and designed the study and wrote the manuscript. KAS, HLR, and DT helped to design, and performed, the experiments. All authors read and approved the final manuscript.

\section{Acknowledgements}

We are grateful to Debbie Marshall for technical assistance with scanning EM and to Ruth Craig (Dartmouth Medical School, Hanover, NH, USA) and Brendan Boyce (University of Rochester Medical Center, Rochester, NY, USA) for helpful discussions. This work was funded by a studentship to KAS from the Arthritis Research Campaign.

\section{References}

1. Rogers MJ: New insights into the molecular mechanisms of action of bisphosphonates. Curr Pharm Des 2003, 9:2643-2658.

2. Frith JC, Monkkonen J, Auriola S, Monkkonen H, Rogers MJ: The molecular mechanism of action of the anti-resorptive and antiinflammatory drug clodronate: evidence for the formation in vivo of a metabolite that inhibits bone resorption and causes osteoclast and macrophage apoptosis. Arthritis Rheum 2001, 44:2201-2210.

3. Coxon FP, Helfrich MH, Van't Hof R, Sebti S, Ralston SH, Hamilton $\mathrm{A}$, Rogers MJ: Protein geranylgeranylation is required for osteoclast formation, function, and survival: inhibition by bisphosphonates and GGTI-298. J Bone Miner Res 2000, 15:1467-1476.

4. Coxon FP, Thompson K, Rogers MJ: Recent advances in understanding the mechanism of action of bisphosphonates. Curr Opin Pharmacol 2006, 6:307-312.

5. Hughes DE, Wright KR, Uy HL, Sasaki A, Yoneda T, Roodman GD, Mundy GR, Boyce BF: Bisphosphonates promote apoptosis in murine osteoclasts in vitro and in vivo. J Bone Miner Res 1995, 10:1478-1487.

6. Xing L, Boyce BF: Regulation of apoptosis in osteoclasts and osteoblastic cells. Biochem Biophys Res Commun 2005, 328:709-720.

7. Asagiri $\mathrm{M}$, Takayanagi $\mathrm{H}$ : The molecular understanding of osteoclast differentiation. Bone 2007, 40:251-264.

8. Schett G, Hayer S, Zwerina J, Redlich K, Smolen JS: Mechanisms of disease: the link between RANKL and arthritic bone disease. Nat Clin Pract Rheumatol 2005, 1:47-54.

9. Takayanagi $\mathrm{H}$ : Osteoimmunological insight into bone damage in rheumatoid arthritis. Mod Rheumato/ 2005, 15:225-231.

10. Teitelbaum SL: Osteoclasts; culprits in inflammatory osteolysis. Arthritis Res Ther 2006, 8:201.

11. Zhao H, Liu S, Huang D, Xu Q, Shuto T, Iwamoto $Y$ : The protective effects of incadronate on inflammation and joint destruction in established rat adjuvant arthritis. Rheumatol Int 2006, 26:732-740.

12. Herrak P, Gortz B, Hayer S, Redlich K, Reiter E, Gasser J, Bergmeister H, Kollias G, Smolen JS, Schett G: Zoledronic acid protects against local and systemic bone loss in tumor necrosis factor-mediated arthritis. Arthritis Rheum 2004, 50:2327-2337.

13. Sims NA, Green JR, Glatt M, Schlict S, Martin TJ, Gillespie MT Romas E: Targeting osteoclasts with zoledronic acid prevents bone destruction in collagen-induced arthritis. Arthritis Rheum 2004, 50:2338-2346.

14. Matsuo $A$, Shuto $T$, Hirata $G$, Satoh $H$, Matsumoto $Y$, Zhao $H$, Iwamoto $Y$ : Antiinflammatory and chondroprotective effects of the aminobisphosphonate incadronate (YM175) in adjuvant induced arthritis. J Rheumatol 2003, 30:1280-1290.

15. Jarrett SJ, Conaghan PG, Sloan VS, Papanastasiou P, Ortmann CE, O'Connor PJ, Grainger AJ, Emery P: Preliminary evidence for a structural benefit of the new bisphosphonate zoledronic acid in early rheumatoid arthritis. Arthritis Rheum 2006, 54:1410-1414.

16. Breuil V, Euller-Ziegler L: Bisphosphonate therapy in rheumatoid arthritis. Joint Bone Spine 2006, 73:349-354.

17. Ritchlin CT, Schwarz EM, O'Keefe RJ, Looney RJ: RANK, RANKL and $O P G$ in inflammatory arthritis and periprosthetic osteolysis. J Musculoskelet Neuronal Interact 2004, 4:276-284.

18. Maksymowych WP: Bisphosphonates for arthritis - a confusing rationale. J Rheumatol 2003, 30:430-434. 
19. Eggelmeijer F, Papapoulos SE, van Paassen HC, Dijkmans BA, Valkema R, Westedt ML, Landman JO, Pauwels EK, Breedveld FC: Increased bone mass with pamidronate treatment in rheumatoid arthritis. Results of a three-year randomized, double-blind trial. Arthritis Rheum 1996, 39:396-402.

20. Coxon FP, Frith JC, Benford HL, Rogers MJ: Isolation and purification of rabbit osteoclasts. In Bone Research Protocols Edited by: Helfrich $\mathrm{MH}$, Ralston SH. Totowa, NJ: Humana Press; 2003:89-99.

21. Benford HL, McGowan NW, Helfrich MH, Nuttall ME, Rogers MJ: Visualization of bisphosphonate-induced caspase- 3 activity in apoptotic osteoclasts in vitro. Bone 2001, 28:465-473.

22. Coxon FP, Helfrich MH, Larijani B, Muzylak M, Dunford JE, Marshall D, McKinnon AD, Nesbitt SA, Horton MA, Seabra MC, Ebetino FH, Rogers MJ: Identification of a novel phosphonocarboxylate inhibitor of Rab geranylgeranyl transferase that specifically prevents Rab prenylation in osteoclasts and macrophages. J Biol Chem 2001, 276:48213-48222.

23. Zhang Q, Badell IR, Schwarz EM, Boulukos KE, Yao Z, Boyce BF, Xing L: Tumor necrosis factor prevents alendronate-induced osteoclast apoptosis in vivo by stimulating $\mathrm{Bcl}-\mathrm{x}_{\mathrm{L}}$ expression through Ets-2. Arthritis Rheum 2005, 52:2708-2718.

24. Monkkonen H, Rogers MJ, Makkonen N, Niva S, Auriola S, Monkkonen J: The cellular uptake and metabolism of clodronate in RAW 264 macrophages. Pharm Res 2001, 18:1550-1555.

25. Lehenkari $P P$, Kellinsalmi $M$, Näpänkangas JP, Ylitalo KV, Mönkkönen J, Rogers MJ, Azhayev A, Väänänen HK, Hassinen IE: Further insight into mechanism of action of clodronate: inhibition of mitochondrial ADP/ATP translocase by a nonhydrolyzable, adenine-containing metabolite. Mol Pharmacol 2002, 61:1255-1262.

26. Roelofs AJ, Thompson K, Gordon S, Rogers MJ: Molecular mechanisms of action of bisphosphonates: current status. Clin Cancer Res 2006, 12:6222s-6230s.

27. Dunford JE, Rogers MJ, Ebetino FH, Phipps RJ, Coxon FP: Inhibition of protein prenylation by bisphosphonates causes sustained activation of Rac, Cdc42 and Rho GTPases. J Bone Miner Res 2006, 21:684-694.

28. Kroemer G, Galluzzi L, Brenner C: Mitochondrial membrane permeabilization in cell death. Physiol Rev 2007, 87:99-163.

29. Eckelman BP, Salvesen GS, Scott FL: Human inhibitor of apoptosis proteins: why XIAP is the black sheep of the family. EMBO Rep 2006, 7:988-994.

30. Karin M, Lin A: NF-kappaB at the crossroads of life and death. Nat Immunol 2002, 3:221-227.

31. Kanaoka K, Kobayashi Y, Hashimoto F, Nakashima T, Shibata M, Kobayashi K, Kato Y, Sakai H: A common downstream signaling activity of osteoclast survival factors that prevent nitric oxidepromoted osteoclast apoptosis. Endocrinology 2000, 141:2995-3005.

32. van Delft MF, Huang DC: How the Bcl-2 family of proteins interact to regulate apoptosis. Cell Res 2006, 16:203-213.

33. Reed JC: Proapoptotic multidomain Bcl-2/Bax-family proteins: mechanisms, physiological roles, and therapeutic opportunities. Cell Death Differ 2006, 13:1378-1386.

34. Kozopas KM, Yang T, Buchan HL, Zhou P, Craig RW: MCL1, a gene expressed in programmed myeloid cell differentiation, has sequence similarity to BCL2. Proc Natl Acad Sci USA 1993, 90:3516-3520.

35. Craig RW: MCL1 provides a window on the role of the BCL2 family in cell proliferation, differentiation and tumorigenesis. Leukemia 2002, 16:444-454.

36. Liu H, Huang Q, Shi B, Eksarko P, Temkin V, Pope RM: Regulation of Mcl-1 expression in rheumatoid arthritis synovial macrophages. Arthritis Rheum 2006, 54:3174-3181.

37. Liu H, Eksarko P, Temkin V, Haines GK 3rd, Perlman H, Koch AE, Thimmapaya B, Pope RM: Mcl-1 is essential for the survival of synovial fibroblasts in rheumatoid arthritis. J Immuno/ 2005 , 175:8337-8345.

38. Smolewska E, Stanczyk J, Robak T, Smolewski P: Inhibited apoptosis of synovial fluid lymphocytes in children with juvenile idiopathic arthritis is associated with increased expression of myeloid cell leukemia 1 and XIAP proteins. J Rheumato/ 2006 , 33:1684-1690.

39. Domina AM, Vrana JA, Gregory MA, Hann SR, Craig RW: MCL1 is phosphorylated in the PEST region and stabilized upon ERK activation in viable cells, and at additional sites with cytotoxic okadaic acid or taxol. Oncogene 2004, 23:5301-5315.

40. Zhou P, Qian L, Kozopas KM, Craig RW: Mcl-1, a Bcl-2 family member, delays the death of hematopoietic cells under a variety of apoptosis-inducing conditions. Blood 1997 89:630-643.

41. Glantschnig $H$, Fisher JE, Wesolowski G, Rodan GA, Reszka AA: M-CSF, TNFalpha and RANK ligand promote osteoclast survival by signaling through mTOR/S6 kinase. Cell Death Differ 2003, 10:1165-1177.

42. Bradley EW, Ruan MM, Oursler MJ: Novel pro-survival functions of the Kruppel-like transcription factor Egr2 in promotion of macrophage colony-stimulating factor-mediated osteoclast survival downstream of the MEK/ERK pathway. $J$ Biol Chem 2008, 283:8055-8064.

43. Donk NW van de, Kamphuis MM, van Kessel B, Lokhorst HM, Bloem AC: Inhibition of protein geranylgeranylation induces apoptosis in myeloma plasma cells by reducing $\mathrm{Mcl}-1$ protein levels. Blood 2003, 102:3354-3362.

44. Suda K, Woo JT, Takami M, Sexton PM, Nagai K: Lipopolysaccharide supports survival and fusion of preosteoclasts independent of TNF-alpha, IL-1, and RANKL. J Cell Physiol 2002, 190:101-108.

45. Oursler MJ, Bradley EW, Elfering SL, Giulivi C: Native, not nitrated, cytochrome $c$ and mitochondria-derived hydrogen peroxide drive osteoclast apoptosis. Am J Physio/ Cell Physiol 2005, 288:C156-C168.

46. Yang-Yen HF: Mcl-1: a highly regulated cell death and survival controller. J Biomed Sci 2006, 13:201-204.

47. Gelinas $\mathrm{C}$, White $\mathrm{E}$ : BH3-only proteins in control: specificity regulates MCL-1 and BAK-mediated apoptosis. Genes Dev 2005, 19:1263-1268.

48. Akiyama T, Bouillet $P$, Miyazaki T, Kadono $Y$, Chikuda $H$, Chung UI, Fukuda A, Hikita A, Seto H, Okada T, Inaba T, Sanjay A, Baron R, Kawaguchi H, Oda H, Nakamura K, Strasser A, Tanaka S: Regulation of osteoclast apoptosis by ubiquitylation of proapoptotic BH3-only Bcl-2 family member Bim. EMBO J 2003 22:6653-6664.

49. Sugatani T, Hruska KA: Akt1/Akt2 and mammalian target of rapamycin/Bim play critical roles in osteoclast differentiation and survival, respectively, whereas Akt is dispensable for cell survival in isolated osteoclast precursors. J Biol Chem 2005, 280:3583-3589.

50. Suyama K, Noguchi Y, Tanaka T, Yoshida T, Shibata T, Saito Y, Tatsuno I: Isoprenoid-independent pathway is involved in apoptosis induced by risedronate, a bisphosphonate, in which Bim plays a critical role in breast cancer cell line MCF-7. Oncol Rep 2007, 18:1291-1298. 